\title{
537.
}

\section{SOLUTIONS OF A SMITH'S PRIZE PAPER FOR 1871.}

[From the Messenger of Mathematics, vol. I. (1872), pp. 37-47, 71-77, 89-95.]

1. A point moves in a plane with a given velocity, and also with a given velocity about a fixed point in the plane: show that the locus is either a circle passing through the fixed point, or else a circle having the fixed point for its centre; and explain the relation between the two solutions.

We have in general

$$
v^{2}=\left(\frac{d r}{d t}\right)^{2}+r^{2}\left(\frac{d \theta}{d t}\right)^{2}
$$

and in the present question, taking the fixed point as the origin, and measuring $\theta$ from any fixed line through this point,

$$
\frac{d \theta}{d t}=\omega, \quad V^{2}=\left(\frac{d r}{d t}\right)^{2}+r^{2} \omega^{2},
$$

where $V, \omega$ are given constants. Hence

$$
\left(\frac{d r}{d \theta}\right)^{2}=\left(\frac{d r}{d t}\right)^{2} \div\left(\frac{d \theta}{d t}\right)^{2}=\frac{V^{2}}{\omega^{2}}-r^{2}
$$

or, writing $V=a \omega$,

$$
\left(\frac{d r}{d \theta}\right)^{2}=a^{2}-r^{2}
$$

therefore

$$
d \theta=\frac{d r}{\left.\sqrt{(} a^{2}-r^{2}\right)},
$$

or

$$
\theta+\beta=\sin ^{-1} \frac{r}{a},(\beta \text { the constant of integration })
$$


that is,

$$
r=a \sin (\theta+\beta),
$$

which is the equation of a circle (radius $=\frac{1}{2} a$ ) passing through the fixed point. In fact, the point moving in such a circle with a constant velocity, moves about the centre with a constant angular velocity, and about any fixed point in the circumference with an angular velocity which is one-half of that about the centre, and is therefore also constant.

Treating $\beta$ as a variable parameter, to obtain the envelope we have

$$
0=a \cos (\theta+\beta),
$$

that is, $\theta+\beta=\frac{\pi}{2}$ and therefore $r=a$, which is the equation of a circle (radius $=a$ ) having the fixed point for its centre. This is consequently the singular solution.

2. Determine the system of curves which satisfy the differential equation

$$
d x\left\{\sqrt{ }\left(1+x^{2}\right)+n y\right\}+d y\left\{\sqrt{ }\left(1+y^{2}\right)+n x\right\}=0 ;
$$

and show that the curve which passes through the point $x=0, y=n$ contains as part of itself the conic

$$
x^{2}+y^{2}+2 x y \sqrt{ }\left(1+n^{2}\right)-n^{2}=0 .
$$

The equation is integrable per se, viz. we have

$$
x \sqrt{ }\left(1+x^{2}\right)+\log \left\{x+\sqrt{ }\left(1+x^{2}\right)\right\}+y \sqrt{ }\left(1+y^{2}\right)+\log \left\{y+\sqrt{ }\left(1+y^{2}\right)\right\}+2 n x y=C,
$$

or, determining the constant so that for $x=0, y$ may be $=n$,

$$
C=n \sqrt{ }\left(1+n^{2}\right)+\log \left\{n+\sqrt{ }\left(1+n^{2}\right)\right\},
$$

and the equation may be written

$$
x \sqrt{ }\left(1+x^{2}\right)+y \sqrt{ }\left(1+y^{2}\right)+2 n x y-n \sqrt{ }\left(1+n^{2}\right)+\log \frac{\left\{x+\sqrt{ }\left(1+x^{2}\right)\right\}\left\{y+\sqrt{ }\left(1+y^{2}\right)\right\}}{n+\sqrt{ }\left(1+n^{2}\right)}=0,
$$

which is evidently a transcendental curve; it may however be shown that, if

$$
x^{2}+y^{2}+2 x y \sqrt{ }\left(1+n^{2}\right)-n^{2}=0,
$$

then we have

and

$$
x \sqrt{ }\left(1+x^{2}\right)+y \sqrt{ }\left(1+y^{2}\right)+2 n x y-n \sqrt{ }\left(1+n^{2}\right)=0,
$$

$$
\left\{x+\sqrt{ }\left(1+x^{2}\right)\right\}\left\{y+\sqrt{ }\left(1+y^{2}\right)\right\}=n+\sqrt{ }\left(1+n^{2}\right),
$$

so that the equation of the curve is thus satisfied; wherefore the transcendental curve contains as part of itself the conic $x^{2}+y^{2}+2 x y \sqrt{ }\left(1+n^{2}\right)-n^{2}=0$.

[As a simple illustration as to how this may happen, take the transcendental curve $y-\sin x y=0$, which it is clear contains as part of itself the line $y=0$.]

C. VIII. 
We have, from the equation of the conic

that is,

$$
\left\{x+y \sqrt{ }\left(1+n^{2}\right)\right\}^{2}=n^{2}\left(1+y^{2}\right) .
$$

$$
x+y \sqrt{ }\left(1+n^{2}\right)= \pm n \sqrt{ }\left(1+y^{2}\right),
$$

but considering the radicals as positive, the sign must be taken so that we have simultaneously $x=0, y=n$. We have therefore

and similarly

$$
x+y \sqrt{ }\left(1+n^{2}\right)=n \sqrt{ }\left(1+y^{2}\right),
$$

Then

$$
y+x \sqrt{ }\left(1+n^{2}\right)=n \sqrt{ }\left(1+x^{2}\right) .
$$

$$
\begin{aligned}
n\left\{x \sqrt{ }\left(1+x^{2}\right)+y \sqrt{ }\left(1+y^{2}\right)\right\} & =2 x y+\left(x^{2}+y^{2}\right) \sqrt{ }\left(1+n^{2}\right) \\
& =2 x y+\sqrt{ }\left(1+n^{2}\right)\left\{n^{2}-2 x y \sqrt{ }\left(1+n^{2}\right)\right\} \\
& =n^{2}\left\{\sqrt{ }\left(1+n^{2}\right)-2 x y\right\}
\end{aligned}
$$

which is the first of the relations in question; and

$$
\begin{aligned}
n^{2}\left\{x+\sqrt{ }\left(1+x^{2}\right)\right\}\{y+ & \left.\sqrt{ }\left(1+y^{2}\right)\right\} \\
= & n^{2} x y+n x\left\{x+y \sqrt{ }\left(1+n^{2}\right)\right\}+n y\left\{y+x \sqrt{ }\left(1+n^{2}\right)\right\} \\
& +x y+\left(x^{2}+y^{2}\right) \sqrt{ }\left(1+n^{2}\right)+x y\left(1+n^{2}\right) \\
= & \left\{n+\sqrt{ }\left(1+n^{2}\right)\right\}\left\{x^{2}+y^{2}+2 x y \sqrt{ }\left(1+n^{2}\right)\right\} \\
= & \left\{n+\sqrt{ }\left(1+n^{2}\right)\right\} n^{2},
\end{aligned}
$$

which is the second of the two relations. And the theorem is thus proved.

[The foregoing is the easiest and most obvious solution, but it is interesting to consider the question differently, as follows:

Write

$$
Q=\frac{\left\{x+\sqrt{ }\left(1+x^{2}\right)\right\}\left\{y+\sqrt{ }\left(1+y^{2}\right)\right\}}{n+\sqrt{ }\left(1+n^{2}\right)},
$$

we have

$$
\begin{aligned}
& Q \quad\left\{\sqrt{ }\left(n^{2}+1\right)+n\right\}=\left\{\sqrt{ }\left(1+x^{2}\right)+x\right\}\left\{\sqrt{ }\left(1+y^{2}\right)+y\right\}=A+B, \\
& Q^{-1}\left\{\sqrt{ }\left(n^{2}+1\right)-n\right\}=\left\{\sqrt{ }\left(1+x^{2}\right)-x\right\}\left\{\sqrt{ }\left(1+y^{2}\right)-y\right\}=A-B,
\end{aligned}
$$

if

$$
\begin{aligned}
& A=\sqrt{ }\left(1+x^{2}\right) \sqrt{ }\left(1+y^{2}\right)+x y, \\
& B=x \sqrt{ }\left(1+y^{2}\right)+y \sqrt{ }\left(1+x^{2}\right) ;
\end{aligned}
$$

and then

$$
\begin{gathered}
A B=x^{2} y \sqrt{ }\left(1+y^{2}\right)+x y^{2} \sqrt{ }\left(1+x^{2}\right) \\
+y\left(1+x^{2}\right) \sqrt{ }\left(1+y^{2}\right)+x\left(1+y^{2}\right) \sqrt{ }\left(1+x^{2}\right) \\
=x \sqrt{ }\left(1+x^{2}\right)+y \sqrt{ }\left(1+y^{2}\right)+2 x y B,
\end{gathered}
$$


that is,

whence

$$
\begin{aligned}
& Q^{2}\left\{2 n^{2}+1+2 n \sqrt{ }\left(1+n^{2}\right)\right\}-\frac{1}{Q^{2}}\left\{2 n^{2}+1-2 n \sqrt{ }\left(1+n^{2}\right)\right\} \\
& =4\left\{x \sqrt{ }\left(1+x^{2}\right)+y \sqrt{ }\left(1+y^{2}\right)\right\} \\
& +4 x y\left[Q\left\{\sqrt{ }\left(1+n^{2}\right)+n\right\}-\frac{1}{Q}\left\{\sqrt{ }\left(1+n^{2}\right)-n\right\}\right],
\end{aligned}
$$

$$
\begin{aligned}
& \text { 4. }\left\{x \sqrt{ }\left(1+x^{2}\right)+y \sqrt{ }\left(1+y^{2}\right)+2 n x y-n \sqrt{ }\left(1+n^{2}\right)\right\} \\
& =Q^{2}\left\{2 n^{2}+1+2 n \sqrt{ }\left(1+n^{2}\right)\right\}-\frac{1}{Q^{2}}\left\{2 n^{2}+1-2 n \sqrt{ }\left(1+n^{2}\right)\right\} \\
& +8 n x y-4 n \sqrt{ }\left(1+n^{2}\right) \\
& -4 x y\left[Q\left\{\sqrt{ }\left(1+n^{2}\right)+n\right\}-\frac{1}{Q}\left\{\sqrt{ }\left(1+n^{2}\right)-n\right\}\right] \\
& =\left(Q^{2}-\frac{1}{Q^{2}}\right)\left(2 n^{2}+1\right)+\left(Q^{2}+\frac{1}{Q^{2}}-2\right) 2 n \sqrt{ }\left(1+n^{2}\right) \\
& -\left(Q-\frac{1}{Q}\right) 4 x y \sqrt{ }\left(1+n^{2}\right)-4\left(Q+\frac{1}{Q}-2\right) n x y \\
& =(Q-1)\left\{\frac{(Q+1)\left(Q^{2}+1\right)}{Q^{2}}\left(2 n^{2}+1\right)\right. \\
& +\frac{(Q-1)(Q+1)^{2}}{Q^{2}} 2 n \sqrt{ }\left(1+n^{2}\right) \\
& -\frac{4(Q+1)}{Q} x y \cdot \sqrt{ }\left(1+n^{2}\right) \\
& \left.-4 \frac{(Q-1)}{Q} n x y\right\} \\
& =(Q-1) \Omega \text { suppose, }
\end{aligned}
$$

that is,

$$
x \sqrt{ }\left(1+x^{2}\right)+y \sqrt{ }\left(1+y^{2}\right)+2 n x y-n \sqrt{ }\left(1+n^{2}\right)=\frac{1}{4}(Q-1) \Omega .
$$

And the integral equation is

$$
\frac{1}{4}(Q-1) \Omega+\log Q=C,
$$

which, for $C=0$, is satisfied by $Q=1$.

Now starting from

we have

$$
Q=\frac{\left\{x+\sqrt{ }\left(1+x^{2}\right)\right\}\left\{y+\sqrt{ }\left(1+y^{2}\right)\right\}}{n+\sqrt{ }\left(1+n^{2}\right)}
$$

$$
\begin{aligned}
& \sqrt{ }\left(1+x^{2}\right)+x=Q\left\{\sqrt{ }\left(1+n^{2}\right)+n\right\}\left\{\sqrt{ }\left(1+y^{2}\right)-y\right\} \\
& \sqrt{ }\left(1+x^{2}\right)-x=\frac{1}{Q}\left\{\sqrt{ }\left(1+n^{2}\right)-n\right\}\left\{\sqrt{ }\left(1+y^{2}\right)+y\right\}
\end{aligned}
$$


and thence

if

$$
2 x=K \sqrt{ }\left(1+y^{2}\right)-L y
$$

$$
\begin{aligned}
& K=Q\left\{\sqrt{ }\left(1+n^{2}\right)+n\right\}-\frac{1}{Q}\left\{\sqrt{ }\left(1+n^{2}\right)-n\right\} \\
& L=Q\left\{\sqrt{ }\left(1+n^{2}\right)+n\right\}+\frac{1}{Q}\left\{\sqrt{ }\left(1+n^{2}\right)-n\right\},
\end{aligned}
$$

wherefore

$$
L^{2}-K^{2}=4
$$

\section{Moreover}

$$
(2 x+L y)^{2}=K^{2}\left(1+y^{2}\right)
$$

that is,

$$
4 x^{2}+\left(L^{2}-K^{2}\right) y^{2}+4 L x y=K^{2}
$$

or, what is the same thing,

$$
x^{2}+y^{2}+L x y=\frac{1}{4}\left(L^{2}-4\right)
$$

which is the rationalised form of

$$
Q=\frac{\left\{x+\sqrt{ }\left(1+x^{2}\right)\right\}\left\{y+\sqrt{ }\left(1+y^{2}\right)\right\}}{n+\sqrt{ }\left(1+n^{2}\right)} .
$$

And if $Q=1$ then $L=2 \sqrt{ }\left(1+n^{2}\right), \frac{1}{4}\left(L^{2}-4\right)=n^{2}$, so that this equation is

$$
x^{2}+y^{2}+2 x y \sqrt{ }\left(1+n^{2}\right)-n^{2}=0 ;
$$

or, when $C=0$, the complete integral is satisfied by

that is, by

$$
\frac{\left\{x+\sqrt{ }\left(1+x^{2}\right)\right\}\left\{y+\sqrt{ }\left(1+y^{2}\right)\right\}}{n+\sqrt{ }\left(1+n^{2}\right)}=1
$$

$$
x^{2}+y^{2}+2 x y \cdot \sqrt{ }\left(1+n^{2}\right)-n^{2}=0 \text {. }
$$

We may without difficulty rationalise, and present the result as follows: the equation

$$
\begin{gathered}
\left\{2\left(x+\frac{1}{x}\right)+\left(n-\frac{1}{n}\right)\left(y-\frac{1}{y}\right)\right\}\left(1+\frac{1}{x^{2}}\right) d x \\
+\left\{2\left(y+\frac{1}{y}\right)+\left(n-\frac{1}{n}\right)\left(x-\frac{1}{x}\right)\right\}\left(1+\frac{1}{y^{2}}\right) d y=0
\end{gathered}
$$

has the complete integral

$$
x^{2}-\frac{1}{x^{2}}+y^{2}-\frac{1}{y^{2}}+\left(x-\frac{1}{x}\right)\left(y-\frac{1}{y}\right)\left(n-\frac{1}{n}\right)-\left(n^{2}-\frac{1}{n^{2}}\right)=C+4 \log \frac{x y}{n},
$$

and a particular integral $x y-n=0$ : the complete integral is in fact

$$
(n-x y)\left\{-n^{3} x^{2} y^{2}+n^{2} x y\left(-x^{2}-y^{2}+1\right)+n\left(x^{2} y^{2}-x^{2}-y^{2}\right)-x y\right\}=x^{2} y^{2} n^{2}\left(C+4 \log \frac{x y}{n}\right),
$$

satisfied, for $C=0$, by $x y-n=0$.] 
3. Write $\alpha=b-c, \beta=c-a, \gamma=a-b$; then considering the three circles and the three conics

$$
\begin{array}{ll}
(x-a)^{2}+y^{2}=-\beta \gamma, & \frac{x^{2}}{b c}+\frac{y^{2}}{K+b c}=1, \\
(x-b)^{2}+y^{2}=-\gamma \alpha, & \frac{x^{2}}{c a}+\frac{y^{2}}{K+c a}=1, \\
(x-c)^{2}+y^{2}=-\alpha \beta, & \frac{x^{2}}{a b}+\frac{y^{2}}{K+a b}=1,
\end{array}
$$

where $K$ is arbitrary; it is required to show that if a variable circle having its centre on one of the conics cuts at right angles the corresponding circle, the envelope is in each of the three cases one and the same bicircular quartic.

Consider the circle $(x-a)^{2}+y^{2}=-\beta \gamma$ and the conic $\frac{x^{2}}{b c}+\frac{y^{2}}{K+b c}=1$, the coordinates of a point on the conic are $\cos \theta \sqrt{ }(b c), \sin \theta \sqrt{ }(K+b c)$, where $\theta$ is a variable parameter; say for a moment these values are $p$ and $q$. The equation of the variable circle is

$$
(x-p)^{2}+(y-q)^{2}=r^{2},
$$

and in order that this may cut at right angles the circle

we must have

$$
(x-a)^{2}+y^{2}=-\beta \gamma,
$$

$$
(p-a)^{2}+q^{2}=r^{2}-\beta \gamma,
$$

or, substituting for $r^{2}$ its value from this equation, the equation of the variable circle is

$$
(x-p)^{2}+(y-q)^{2}=(a-p)^{2}+q^{2}+\beta \gamma,
$$

that is,

viz. this is

$$
x^{2}+y^{2}-a^{2}-\beta \gamma-2 p(x-a)-2 q y=0,
$$

$$
\left(x^{2}+y^{2}-a^{2}-\beta \gamma\right)-2(x-a) \sqrt{ }(b c) \cos \theta-2 y \sqrt{ }(K+b c) \sin \theta=0 .
$$

Hence taking the envelope in regard to $\theta$, the equation is

$$
\left(x^{2}+y^{2}-a^{2}-\beta \gamma\right)^{2}-4(x-a)^{2} b c-4 y^{2}(K+b c)=0,
$$

that is,

$$
\left(x^{2}+y^{2}-a b-a c+b c\right)^{2}-4(x-a)^{2} b c-4 y^{2}(K+b c)=0,
$$

or, what is the same thing,

$$
\begin{aligned}
\left(x^{2}+y^{2}\right)^{2}-2(b c & +c a+a b)\left(x^{2}+y^{2}\right)-4 K y^{2}+8 a b c x \\
& +b^{2} c^{2}+c^{2} a^{2}+a^{2} b^{2}-2 a^{2} b c-2 b^{2} c a-2 c^{2} a b=0,
\end{aligned}
$$

viz. this equation, being symmetrical in regard to $a, b, c$, is the same equation as would have been obtained from either of the other conics and the corresponding circle; and from the form of the equation it is clear that the curve is a bicircular quartic. 
4. Show that the caustic by refraction for parallel rays of a circle, radius $c$, index of refraction $\mu$, is the same curve as the caustic by refraction for parallel rays of the concentric circle, radius $\frac{c}{\mu}$, index of refraction $\frac{1}{\mu}$.

Take as usual $\mu>1$. Imagine the ray $A P$ (fig. 1) parallel to the axis of $x$, incident at $P$ on the circle radius $c$, and let the refracted ray after cutting the circle radius $\frac{c}{\mu}$, cut it again in $Q$, and then cut the axis in $R$. Take $\phi, \phi^{\prime}$ for the angles of incidence and refraction; $\sin \phi=\mu \sin \phi^{\prime}$.

Fig. 1.

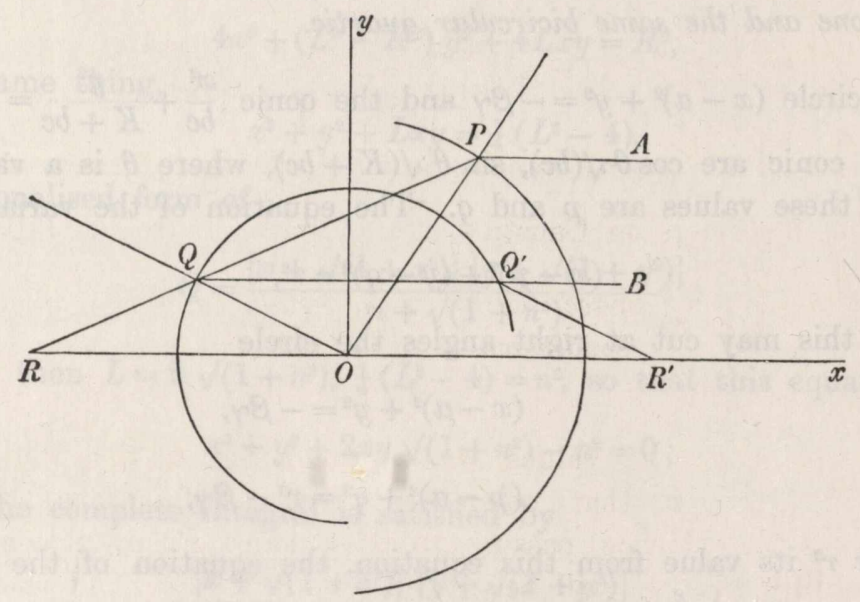

Moreover in the triangle $P Q O$, we have $\sin Q: \sin P=c: \frac{c}{\mu}$; that is, $\sin Q=\mu \sin P$, $=\mu \sin \phi^{\prime},=\sin \phi$; or $\angle Q=\phi$. And then in the triangle $R Q O, \angle R=\phi-\phi^{\prime}, \angle Q=180^{\circ}-\phi$, whence $\angle O=\phi^{\prime}$, that is, $\angle Q O R=\phi^{\prime}$.

Consider now a ray $B Q$ incident at $Q$ and refracted in the direction $Q R$; the index of refraction being $\frac{1}{\mu}$, that is, the denser medium being on the outside of the small circle. Taking $\theta, \theta^{\prime}$ for the angles of incidence and refraction, we have $\sin \theta=\frac{1}{\mu} \sin \theta^{\prime}$; but, the refracted ray being by hypothesis $Q R$, we have by what precedes $\theta^{\prime}=\phi$, hence $\sin \theta=\frac{1}{\mu} \sin \phi=\sin \phi^{\prime}$, that is, $\theta=\phi^{\prime}$, or $\angle B Q O=\angle Q O R$, that is, the incident ray $B Q$ is parallel to the axis of $x$. We have thus two pencils of rays each parallel to the axis, such that for any ray $A P$ of the first pencil there is a corresponding ray $B Q$ of the second pencil, the rays $A P$ and $B Q$ each giving rise to the same refracted ray $P Q R$; hence the two pencils have the same caustic.

[It is proper to remark that for the ray $B Q$ it has been assumed that the refraction takes place not at $Q^{\prime}$ where it first meets the small circle, but at $Q$; if 
we consider the refraction at $Q^{\prime}$, then the index of refraction is still to be $=\frac{1}{\mu}$, that is, the denser medium must now be inside the small circle; the refracted ray is in the direction $R^{\prime} Q^{\prime}$ situate symmetrically with $R Q$ on the opposite side of the axis of $y$; and it would at first sight appear that the caustic was a curve equal and similar to the original caustic, but situate on the opposite side of the axis of $y$. But geometrically the complete caustic consists of two equal and similar portions situate on opposite sides of the axis of $y$; so that we really obtain, not an equal and opposite caustic, but in each case one and the same caustic.

I originally obtained the theorem in a different manner; viz. the equation for the caustic for the first pencil of rays was found to be

$$
\left(1-\mu^{2}\right) \frac{x}{c}=\left\{1-\mu^{\frac{4}{3}}\left(\frac{y}{c}\right)^{\frac{2}{3}}\right\}^{\frac{2}{3}}+\mu\left\{1-\mu^{-\frac{2}{3}}\left(\frac{y}{c}\right)^{\frac{2}{3}}\right\}^{\frac{3}{2}}
$$

which equation (as is easily seen) remains unaltered when $c, \mu$ are changed into $\frac{c}{\mu}, \frac{1}{\mu}$ respectively.-See my "Memoir on Caustics," Phil. Trans., t. CXLVII. (1857), [145], p. 285.]

5. Given at each point of space the direction-cosines $(\alpha, \beta, \gamma)$ of a line through that point: it is required to find the conditions in order that the lines may be not a triple but a double system.

For any given point $P$ the values of the quantities $\alpha, \beta, \gamma$ which determine the direction of the line through that point are given as functions of the coordinates $(x, y, z)$ of the point $P$. Hence passing from a point $P$ to a consecutive point $P^{\prime}$ on the line, the coordinates of $P^{\prime}$ will be $x+\rho \alpha, y+\rho \beta, z+\rho \gamma$; and the values of $\alpha, \beta, \gamma$ for the point $P^{\prime}$ will be

$$
\begin{aligned}
& \alpha+\rho\left(\alpha \frac{d \alpha}{d x}+\beta \frac{d \alpha}{d y}+\gamma \frac{d \alpha}{d z}\right) \\
& \beta+\rho\left(\alpha \frac{d \beta}{d x}+\beta \frac{d \beta}{d y}+\gamma \frac{d \beta}{d z}\right), \\
& \gamma+\rho\left(\alpha \frac{d \gamma}{d x}+\beta \frac{d \gamma}{d y}+\gamma \frac{d \gamma}{d z}\right)
\end{aligned}
$$

But if the lines form a double system, we must have the same line for the point $P$, and for any other point $P^{\prime}$ on the line; and in particular the same line for the point $P$, and for the consecutive point $P^{\prime}$. Hence as conditions for the double system we obtain

$$
\begin{aligned}
& \alpha \frac{d \alpha}{d x}+\beta \frac{d \alpha}{d y}+\gamma \frac{d \alpha}{d z}=0 \\
& \alpha \frac{d \beta}{d x}+\beta \frac{d \beta}{d y}+\gamma \frac{d \beta}{d z}=0 \\
& \alpha \frac{d \gamma}{d x}+\beta \frac{d \gamma}{d y}+\gamma \frac{d \gamma}{d z}=0
\end{aligned}
$$


But in virtue of the relation $\alpha^{2}+\beta^{2}+\gamma^{2}=1$, we have

$$
\begin{aligned}
& \alpha \frac{d \alpha}{d x}+\beta \frac{d \beta}{d x}+\gamma \frac{d \gamma}{d x}=0 \\
& \alpha \frac{d \alpha}{d y}+\beta \frac{d \beta}{d y}+\gamma \frac{d \gamma}{d y}=0 \\
& \alpha \frac{d \alpha}{d z}+\beta \frac{d \beta}{d z}+\gamma \frac{d \gamma}{d z}=0 .
\end{aligned}
$$

Hence subtracting the corresponding equations we have three equations, which are ato. once seen to be equivalent to the two equations

$$
\frac{d \beta}{d z}-\frac{d \gamma}{d y}: \frac{d \gamma}{d x}-\frac{d \alpha}{d z}: \frac{d \alpha}{d y}-\frac{d \beta}{d x}=\alpha: \beta: \gamma
$$

equations which must be satisfied identically, whatever are the values of $(x, y, z)$. The equations have been obtained as necessary conditions; they are, in fact, the sufficient conditions for a double system; for the line being unaltered in passing from $P$ to $P^{\prime}$, it remains unaltered when we pass to the following point $P^{\prime \prime}$, and so on ; that is, for the passage to any point $Q$ whatever on the line.

CoR. If the equation $\alpha d x+\beta d y+\gamma d z=0$ be integrable by a factor, it must be integrable per se: in fact, the condition that it may be integrable by a factor is

$$
\alpha\left(\frac{d \beta}{d z}-\frac{d \gamma}{d y}\right)+\beta\left(\frac{d \gamma}{d x}-\frac{d \alpha}{d z}\right)+\gamma\left(\frac{d \alpha}{d y}-\frac{d \beta}{d x}\right)=0
$$

But we have

$$
\frac{d \beta}{d z}-\frac{d \boldsymbol{\gamma}}{d y}=k \alpha, \& \bar{c}
$$

and the equation thus becomes

$$
k\left(\alpha^{2}+\beta^{2}+\gamma^{2}\right)=0,
$$

that is, $k=0$, and therefore

$$
\frac{d \beta}{d z}-\frac{d \gamma}{d y}=0, \quad \frac{d \gamma}{d x}-\frac{d \alpha}{d z}=0, \quad \frac{d \alpha}{d y}-\frac{d \beta}{d x}=0
$$

Hence, also, if the lines cut at right angles a surface, we must have $\alpha d x+\beta d y+\gamma d z$ a complete differential.

The foregoing theory is given in Sir W. R. Hamilton's "Memoir on Ray-Systems."

6. If $X=0, Y=0, Z=0, W=0$ are four given conics in the same plane and having a common point: show that, in the system of conics $a X+b Y+c Z+d W=0$, there are in general four (improper) conics the equations of which may be taken to be $x^{2}=0, y^{2}=0, x z=0, y z=0$. 
Taking the conics to pass through the point $x=0, y=0$; their equations will be of the form

$$
\begin{aligned}
& X=a_{1} x^{2}+2 h_{1} x y+b_{1} y^{2}+2 f_{1} y z+2 g_{1} z x=0 \\
& Y=a_{2} x^{2}+2 h_{2} x y+b_{2} y^{2}+2 f_{2} y z+2 g_{2} z x=0 \\
& Z=a_{3} x^{2}+2 h_{3} x y+b_{3} y^{2}+2 f_{3} y z+2 g_{3} z x=0 \\
& W=a_{4} x^{2}+2 h_{4} x y+b_{4} y^{2}+2 f_{4} y z+2 g_{4} z x=0
\end{aligned}
$$

Now multiplying by the indeterminate quantities $\alpha, \beta, \gamma, \delta$, the three ratios $\alpha: \beta: \gamma: \delta$ may be determined so that the terms in $y z, z x$ shall vanish, and the terms in $x^{2}, x y, y^{2}$ be a perfect square: we thus arrive at a quadric equation for any one of the ratios, say $\alpha: \beta$, the remaining ratios being then linearly determined; viz. there are two sets of values of $\alpha, \beta, \gamma, \delta$ : and changing the coordinates $(x, y)$, the two resulting forms may be represented by $x^{2}=0, y^{2}=0$.

And it is clear that we thus have in the system of conics $\alpha X+\beta Y+\gamma Z+\delta W=0$, four conics the equations of which may be represented by

$$
\begin{array}{lr}
X^{\prime}=x^{2} & =0, \\
Y^{\prime} & =y^{2} \\
Z^{\prime} & =h_{3} x y+f_{3} y z+g_{3} z x=0, \\
W^{\prime}=h_{4} x y+f_{4} y z+g_{4} z x & =0,
\end{array}
$$

where of course the coefficients $f, g, h$ have new values.

We may then form the equations

$$
\begin{aligned}
& \alpha X^{\prime}+f_{4} Z^{\prime}-f_{3} W^{\prime}=x\left\{\alpha x+\left(f_{4} h_{3}-f_{3} h_{4}\right) y+\left(f_{4} g_{3}-f_{3} g_{4}\right) z\right\}, \\
& \beta Y^{\prime}-g_{4} Z^{\prime}+g_{3} W^{\prime}=y\left\{\left(g_{3} h_{4}-g_{4} h_{3}\right) x+\beta y+\left(f_{4} g_{3}-f_{3} g_{4}\right) z\right\},
\end{aligned}
$$

so that, by writing $\alpha=g_{3} h_{4}-g_{4} h_{3}$ and $\beta=f_{4} h_{3}-f_{3} h_{4}$, the terms in \{\} will be one and the same linear function of $(x, y, z)$; that is, changing the $z$ so as to denote the linear function in question by $z$, we have as conics of the series $x z=0$, and $y z=0$, that is, we have in the series the four conics $x^{2}=0, y^{2}=0, x z=0, y z=0$; whence also any other conic of the series, and consequently each of the original four conics, may be represented by an equation of the form

$$
a x^{2}+b y^{2}+2 f y z+2 g z x=0 .
$$

7. The coordinates $(x, y, z, w)$ of a point $P$ in space are connected with the coordinates $\left(x^{\prime}, y^{\prime}, z^{\prime}\right)$ of a point $P^{\prime}$ in a plane by the equations

$$
x: y: z: w=X^{\prime}: Y^{\prime}: Z^{\prime}: W^{\prime},
$$

where $X^{\prime}, Y^{\prime}, Z^{\prime}, W^{\prime}$ are quadric functions of $\left(x^{\prime}, y^{\prime}, z^{\prime}\right)$ such that $X^{\prime}=0, Y^{\prime}=0, Z^{\prime}=0$, $W^{\prime}=0$ represent conics having a common point: show that the locus of $P$ is a cubic scroll (skew surface of the third order): and find the curves in the plane which correspond to the generating lines of the scroll.

C. VIII. 
The equations $x: y: z: w=X^{\prime}: Y^{\prime}: Z^{\prime}: W^{\prime}$ are three equations containing the indeterminate parameters $x^{\prime}: z^{\prime}$ and $y^{\prime}: z^{\prime}$, so that eliminating these we have between $(x, y, z, w)$ a single (homogeneous) equation representing a surface. To each point $\left(x^{\prime}, y^{\prime}, z^{\prime}\right)$ of the plane, there corresponds a single point of the surface, and to each point $(x, y, z, w)$ of the surface a single point $\left(x^{\prime}, y^{\prime}, z^{\prime}\right)$ of the plane. The only exception is that for the common point of the four conics, the ratios $x: y: z: w$ are essentially indeterminate, and there is not corresponding hereto any determinate point of the surface.

To find the order of the surface, consider its intersection with any arbitrary line

$$
\begin{aligned}
& a x+b y+c z+d w=0, \\
& a_{1} x+b_{1} y+c_{1} z+d_{1} w=0 .
\end{aligned}
$$

We have corresponding hereto in the plane the points of intersection of the conics

$$
\begin{aligned}
& a X^{\prime}+b Y^{\prime}+c Z^{\prime}+d W^{\prime}=0, \\
& a_{1} X^{\prime}+b_{1} Y^{\prime}+c_{1} Z^{\prime}+d_{1} W^{\prime}=0,
\end{aligned}
$$

viz. these are conics each of them passing through the common point of the four conics, and therefore they intersect besides in three points: that is, the order of the surface is $=3$.

To show that the common point ought to be (as above) excluded, some further explanation is desirable. To the section of the surface by the plane $a x+b y+c z+d w=0$, corresponds the conic $a X^{\prime}+b Y^{\prime}+c Z^{\prime}+d W^{\prime}=0$; and similarly to the section by the plane $a_{1} x+b_{1} y+c_{1} z+d_{1} w=0$, corresponds the conic $a_{1} X^{\prime}+b_{1} Y^{\prime}+c_{1} Z^{\prime}+d_{1} W^{\prime}=0$. Now to the common point considered as belonging to the first conic there corresponds a determinate point of the surface; and to the common point considered as belonging to the second conic there corresponds a determinate point of the surface; but these are two distinct points on the surface: so that corresponding to the common point of the four conics, there is not on the surface any point of intersection of the two plane sections; but these intersect in only three points of the surface; viz. the line of intersection of the two planes meets the surface in three points: or the surface is a cubic surface.

The same result may be obtained, and it may be further shown that the surface is a scroll, by means of the property in the foregoing question 6 ; viz it thereby appears that each of the functions $X^{\prime}, Y^{\prime}, Z^{\prime}, W^{\prime}$ may be taken to be of the form $a x^{\prime 2}+b y^{\prime 2}+f y^{\prime} z^{\prime}+g z^{\prime} x^{\prime}$; hence replacing the original coordinates $x, y, z, w$, by properly selected linear functions of these coordinates, the given relations may be presented in the form

$$
x: y: z: w=x^{\prime 2}: y^{\prime 2}: x^{\prime} z^{\prime}: y^{\prime} z^{\prime},
$$

whence eliminating, we have

$$
x w^{2}-y z^{2}=0
$$

the equation of a cubic scroll, having the line $z=0, w=0$ for a double line, and the line $x=0, y=0$ for a directrix line. The equations of a generating line of the scroll 
are, it is clear, $z-\theta w=0, x-\theta^{2} y=0$, where $\theta$ is a variable parameter; and corresponding hereto in the plane we have the line $x^{\prime}-\theta y^{\prime}=0$, viz. this is any line through the common intersection of the four conics.

8. If $U, V$ are binary functions of the form $(a, b, \ldots)(x, y)^{m}$ with arbitrary coefficients, and if the equations $U=0, V=0$ have a common root, show how this can be determined in terms of the derived functions of the Resultant in regard to the coefficients of either function.

Show what results in regard to the common root can be obtained when the coefficients are not all of them arbitrary but (1) each or either of the functions depends in any manner whatever on a set of arbitrary coefficients not entering into the other function, (2) the two functions depend in any manner whatever on one and the same set of arbitrary coefficients.

How is the theory modified when, instead of the two equations, there is a single equation $U=0$ having a double root?

Suppose

$$
\begin{aligned}
& U=(a, b, \ldots)(x, y)^{m}\left(=a x^{m}+\frac{m}{1} b x^{m-1} y+\& c .\right) \\
& V=\left(a^{\prime}, b^{\prime}, \ldots\right)(x, y)^{m^{\prime}}\left(=a^{\prime} x^{m^{\prime}}+\frac{m^{\prime}}{1} b x^{m^{\prime}-1} y+\& c .\right) .
\end{aligned}
$$

Then if $R$ is the resultant, the equation $R=0$ is the relation which must exist between the coefficients $(a, b, \ldots)$ and $\left(a^{\prime}, b^{\prime}, \ldots\right)$ in order that the equations $U=0$ and $V=0$ may have a common root (that is, in order that the functions $U, V$ may have a common factor $x-\alpha y$ ). Imagine the relation subsisting, and that $x, y$ are the values belonging to the common root, or (what is the same thing) that we have $x-\alpha y=0$; we have then simultaneously $U=0, V=0, R=0$. Now suppose the coefficients $a, b, \ldots$ to be infinitesimally varied in such manner that $U, V$ have still a common root; say the new values are $a+\delta a, b+\delta b, \ldots$ : this implies between $\delta a, \delta b, \ldots$ the relation

$$
\frac{d R}{d a} \delta a+\frac{d R}{d b} \delta b+\ldots=0
$$

But the common factor $x-\alpha y$ is a factor of the unaltered equation $V=0$; and the values of $(x, y)$ are thus unaltered, viz. the equation $U=0$ is satisfied with the original values of $(x, y)$; so that we have

$$
\frac{d U}{d a} \delta a+\frac{d U}{d b} \delta b+\ldots=0
$$

or, what is the same thing,

$$
x^{m} \delta a+m x^{m-1} y \delta b+\ldots=0,
$$

an equation which must agree with the former one, that is, we have

$$
x^{m}: m x^{m-1} y: \& c .=\frac{d R}{d a}: \frac{d R}{d b}: \& c .,
$$


a series of equations giving the value of the common root $\frac{x}{y}(=\alpha)$ in the several forms

$$
\frac{1}{m} \frac{x}{y}=\frac{d R}{d a} \div \frac{d R}{d b}, \quad \frac{2}{m-1} \frac{x}{y}=\frac{d R}{d b} \div \frac{d R}{d c}, \text { \&c. }
$$

And it is clear that we have in like manner

$$
x^{m^{\prime}}: m^{\prime} x^{m^{\prime}-1} y: \& \mathrm{c} .=\frac{d R}{d a^{\prime}}: \frac{d R}{d b^{\prime}}: \& c .
$$

It is clear that if $U$ involves, in any manner whatever, the coefficients $a, b, \ldots$ which do not enter into the function $V$, then we have in precisely the same manner

$$
\frac{d U}{d a}: \frac{d U}{d b}: \& c .=\frac{d R}{d a}: \frac{d R}{d b}: \& c .
$$

a system of equations satisfied by the values $x, y$ which belong to the common root.

But if the coefficients $a, b, \ldots$ are contained in any manner whatever in both of the functions $U, V$; then by altering $a, b, \ldots$ we alter the common root; say that $x+\delta x, y+\delta y$ belong to its new value; then we have

$$
\begin{aligned}
& \frac{d U}{d x} \delta x+\frac{d U}{d y} \delta y+\frac{d U}{d a} \delta a+\frac{d U}{d b} \delta b+\ldots=0 \\
& \frac{d V}{d x} \delta x+\frac{d V}{d y} \delta y+\frac{d V}{d a} \delta a+\frac{d V}{d b} \delta b+\ldots=0 .
\end{aligned}
$$

Now the values of $x, y$ which satisfy $U=0, V=0$ also satisfy

$$
\frac{d U}{d x} \frac{d V}{d y}-\frac{d U}{d y} \frac{d V}{d x}=0
$$

hence from the foregoing two equations eliminating $\delta x$ or $\delta y$, the other of these two quantities will disappear of itself, and we thus obtain an equation

$$
A \delta a+B \delta b+\ldots=0
$$

which must agree with the above equation

or we have

$$
\frac{d R}{d a} \delta a+\frac{d R}{d b} \delta b+\ldots=0
$$

$$
\frac{d R}{d a}: \frac{d R}{d b}: \& c .=A: B: \& c .,
$$

a system of equations satisfied by the values $x, y$ which belong to the common root.

In the case of a single equation $U=0$ having a double root, the condition for this is $\Delta=0$, where $\Delta$ is the discriminant of the function $U$; and the like reasoning shows that for the values $x, y$ which belong to the double root we have

$$
\frac{d U}{d a}: \frac{d U}{d b}: \& c .=\frac{d \Delta}{d a}: \frac{d \Delta}{d b}: \ldots ;
$$


viz. if $U$ is of the form $(a, b, \ldots)(x, y)^{m}$ with arbitrary coefficients, then we have thus a series of equations giving the required value of $\frac{x}{y}$; but if $(a, b, \ldots)$ are arbitrary coefficients contained in any manner whatever in the function $U$, then we have a series of equations satisfied by the values $x, y$ which belong to the double root.

9. The normal at each point of a principal section of an ellipsoid is intersected by the normal at a consecutive point not on the principal section: show that the locus of the point of intersection is an ellipse having four (real or imaginary) contacts with the evolute of the principal section.

The principal section is for convenience taken to be that in the plane of $z x$; the coordinates of any point thereof are therefore $X, 0, Z$ where

$$
\frac{X^{2}}{a^{2}}+\frac{Z^{2}}{c^{2}}=1
$$

Consider the normal at a point $X, Y, Z$ of the ellipsoid; taking $x, y, z$ as current coordinates, the equations of the normal are

$$
\frac{x-X}{\frac{X}{a^{2}}}=\frac{y-Y}{\frac{Y}{b^{2}}}=\frac{z-Z}{\frac{Z}{c^{2}}} .
$$

Writing herein $y=0$, we have

$$
x=X\left(1-\frac{b^{2}}{a^{2}}\right), \quad z=Z\left(1-\frac{b^{2}}{c^{2}}\right) ;
$$

viz. $x, z$ are here the coordinates of the point where the normal meets the plane of $x z$; and observing that the point in question lies on the normal at the point $X, 0, Z$, it is clear that $x, y, z$ will be the coordinates of the intersection of the last-mentioned normal by the normal at the consecutive point not on the principal section.

Writing for shortness

$$
\alpha=b^{2}-c^{2}, \quad \beta=c^{2}-a^{2}, \quad \gamma=a^{2}-b^{2},
$$

$(\alpha+\beta+\gamma=0, \alpha$ and $\gamma$ positive, $\beta$ negative) the values are

wherefore

$$
x=\frac{\gamma X}{a^{2}}, \quad z=-\frac{\alpha Z}{c^{2}},
$$

or, substituting in

$$
\frac{X}{a}=\frac{a x}{\gamma}, \quad \frac{Z}{c}=-\frac{c z}{x}
$$

$$
\frac{X^{2}}{a^{2}}+\frac{Z^{2}}{c^{2}}=1
$$


we have

$$
\frac{a^{2} x^{2}}{\gamma^{2}}+\frac{c^{2} z^{2}}{\alpha^{2}}=1
$$

the required locus, which is thus an ellipse.

If the point $(X, 0, Z)$ is an umbilicus, it is clear that the corresponding point of the locus will be a point of the evolute of the principal section; and to prove that the locus touches the evolute, it is only necessary to show that the tangent of the locus is also the tangent of the evolute; or what is the same thing, that the tangent of the locus passes through the umbilicus.

Now for the umbilicus we have

$$
X^{2}=-a^{2} \frac{\gamma}{\beta}, \quad Z^{2}=-c^{2} \frac{\alpha}{\beta}
$$

the corresponding values of $x, z$ being

$$
x=\frac{\gamma X}{a^{2}}, \quad z=-\frac{\alpha Z}{c^{2}} .
$$

Take $\xi, \zeta$ as the current coordinates of a point on the tangent of the locus, we have

$$
\frac{a^{2} x \xi}{\gamma^{2}}+\frac{c^{2} z \zeta}{\alpha^{2}}=1
$$

or, substituting for $x, z$ the foregoing values,

$$
\frac{X \xi}{\gamma}-\frac{Z \zeta}{a}=1
$$

and these should be satisfied by $\xi, \zeta=X, Z$; viz. we ought to have

$$
\frac{X^{2}}{\gamma}-\frac{Z^{2}}{\alpha}=1
$$

and this equation is in fact true for the values of $X, Z$ at the umbilicus; viz. for these values we have

$$
-\frac{a^{2}}{\beta}+\frac{c^{2}}{\beta}=1
$$

that is, $\beta=c^{2}-\alpha^{2}$, which is in fact the value of $\beta$.

There is obviously a contact in each quadrant, that is, there are four contacts (in the present case all real) of the locus with the evolute.

The same theorem holds good in regard to the other principal sections; only for these, the umbilici being imaginary, the points of contact of the locus with the evolute are also imaginary.

Remark. There is a great convenience in questions relating to the ellipsoid, in the use of the foregoing notations $\alpha, \beta, \gamma$. 
10. An endless heavy chain of given length is suspended from two fixed points in the same horizontal plane: show that (subject to a condition as to the length) the figure of equilibrium may consist of portions of two distinct catenaries.

The two parts of the chain will each of them be a portion of a catenary, viz. they will either coincide with each other, forming a twice repeated portion of a catenary (which is always a possible position of equilibrium), or they will form portions of two distinct catenaries. That the latter form is in some cases possible, appears from the case of a very long chain. It is then clear that there is a position of equilibrium in which the upper catenary is nearly a straight line. It may be added, that, as the length of the chain diminishes, the two distinct catenaries approach more and more, and for a certain value of the length become coincident; for any smaller value of the length, the only position is that consisting of a twice repeated portion of a catenary. But to obtain the solution in a regular manner, observe that, in order to the existence of such a form of equilibrium, the necessary condition is, that the tension at $A$ (or $B$ ) must be equal in the two catenaries. Now the tension at any point of a catenary is proportional to the height above the directrix of the catenary; hence the condition is, that there shall be through the points $A, B$ two catenaries having the same directrix, and such that the sum of the lengths is equal to the given length of the chain.

Take $A B=2 a$, the length of the chain $=2 l$. Take $\beta$ for the distance of the directrix below the points $A, B ; c$ for the parameter of the catenary (or distance of its lowest point above the directrix), $\beta, c$ being of course unknown. Then taking the origin at the mid-point of the directrix, and the axis of $y$ vertically upwards, the equation of the catenary is

$$
y=\frac{c}{2}\left(e^{\frac{x}{c}}+e^{-\frac{x}{c}}\right)
$$

whence for the point $A$ or $B$,

$$
\beta=\frac{c}{2}\left(e^{\frac{a}{c}}+e^{-\frac{a}{c}}\right)
$$

and the arc measured from the lowest point is

$$
s=\frac{c}{2}\left(e^{-\frac{x}{c}}-e^{-\frac{x}{c}}\right) .
$$

Hence, assuming that there are two distinct catenaries, if the parameters are $c$, $c^{\prime}$, we have

$$
\begin{aligned}
& \frac{1}{2} c\left(e^{\frac{a}{c}}+e^{-\frac{a}{c}}\right)=\frac{1}{2} c^{\prime}\left(e^{\frac{a}{c^{\prime}}}+e^{-\frac{a}{c^{\prime}}}\right), \\
& \frac{1}{2} c\left(e^{\frac{a}{c}}-e^{-\frac{a}{c}}\right)+\frac{1}{2} c^{\prime}\left(e^{\frac{a}{c^{\prime}}}-e^{-\frac{a}{c^{\prime}}}\right)=l,
\end{aligned}
$$

which are the conditions for the determination of $c, c^{\prime}$; and it is to be shown that these can be satisfied otherwise than by taking $c=c^{\prime}$. 
Trace the two curves

$$
\begin{aligned}
& y=\frac{x}{2}\left(e^{\frac{a}{x}}+e^{-\frac{a}{x}}\right) \\
& y^{\prime}=\frac{x}{2}\left(e^{\frac{a}{x}}-e^{-\frac{a}{x}}\right)
\end{aligned}
$$

shown respectively by the black line and the dotted line in fig. 2. Draw any line parallel to the axis of $x$, meeting the first curve in the points $P, P^{\prime}$ respectively, and let the ordinates $M P, M^{\prime} P^{\prime}$ meet the second curve in the points $Q, Q^{\prime}$ respectively;

Fig. 2.

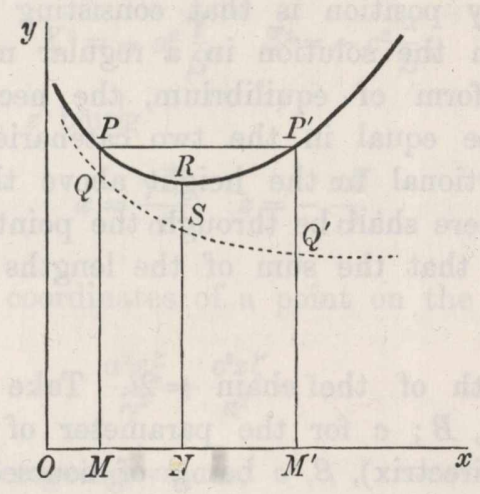

then it is clear, that if for a given value of $l$ the line $P P^{\prime}$ can be drawn in suchwise that $M Q+M^{\prime} Q^{\prime}=l$, there will be in fact the required two values $c=O M$ and $c^{\prime}=O M^{\prime}$.

And since for $M P$ very large we have $M Q$, and therefore also $M Q+M^{\prime} Q^{\prime}$, very large, and as $M P$ diminishes, $M Q+M^{\prime} Q^{\prime}$ also diminishes until it attains a certain minimum value, say $=\lambda$, it is clear that if $l$ has any value greater than this minimum value, $P P^{\prime}$ can be so drawn that $Q M+Q^{\prime} M^{\prime}=l$.

['The above remarkably elegant investigation in regard to the two values $c, c^{\prime}$ was given in the Examination; it seems to be the case that as $P P^{\prime}$ moves downwards, $M Q+M^{\prime} Q^{\prime}$ continually decreases (viz. $M Q$ decreases more rapidly than $M^{\prime} Q^{\prime}$ increases), its value being least, and $=2 N S$ when $P P^{\prime}$ becomes a tangent to the first curve at its lowest point $R$; but it is not by any means easy to prove that this is so. The question depends on the form of the curve defined by the equations

$$
\begin{aligned}
& X=\frac{1}{2} x_{1}\left(e^{\frac{a}{x_{1}}}-e^{-\frac{a}{x_{1}}}\right)+\frac{1}{2} x_{2}\left(e^{\frac{a}{x_{2}}}-e^{-\frac{a}{x_{2}}}\right), \\
& Y=\frac{1}{2} x_{1}\left(e^{\frac{a}{x_{2}}}+e^{-\frac{a}{x_{2}}}\right)=\frac{1}{2} x_{2}\left(e^{\frac{a}{x_{2}}}+e^{-\frac{a}{x_{2}}}\right)
\end{aligned}
$$

where $X$ and $Y$ are the current coordinates.] 
11. A particle is attracted to two centres of force, one of them at the origin, the other revolving about the origin in a circle in the plane of xy with a uniform angular velocity $n^{\prime}$ : find the equations of motion; and writing $v$ for the velocity of the particle and $A$ for the resolved area (about the fixed centre) in the plane of $x y$, show that there is a first integral giving the value of $v^{2}-4 n^{\prime} \frac{d A}{d t}$ in terms of the coordinates of the particle and of the revolving centre.

Take $\xi, \eta, 0$ for the coordinates of the moving centre, so that

the equations of motion are

$$
\xi=a \cos n^{\prime} t, \quad \cdot \eta=a \sin n^{\prime} t ;
$$

$$
\begin{aligned}
& \frac{d^{2} x}{d t^{2}}=-\phi r \frac{x}{r}-\psi \rho \frac{x-\xi}{\rho}, \\
& \frac{d^{2} y}{d t^{2}}=-\phi r \frac{y}{r}-\psi \rho \frac{y-\eta}{\rho}, \\
& \frac{d^{2} z}{d t^{2}}=-\phi r \frac{z}{r}-\psi \rho \frac{z}{\rho},
\end{aligned}
$$

where

We have

$$
\begin{aligned}
& r^{2}=x^{2}+y^{2}+z^{2}, \\
& \rho^{2}=(x-\xi)^{2}+(y-\eta)^{2}+z^{2} .
\end{aligned}
$$

$$
\begin{aligned}
& r d r=x d x+y d y+z d z, \\
& \rho d \rho=(x-\xi)(d x-d \xi)+(y-\eta)(d y-d \eta)+z d z .
\end{aligned}
$$

But

whence

$$
\begin{aligned}
& d \xi=-n^{\prime} a \sin n^{\prime} t d t=-n^{\prime} \eta d t, \\
& d \eta=n^{\prime} a \cos n^{\prime} t d t=n^{\prime} \xi d t,
\end{aligned}
$$

$$
\begin{aligned}
\rho d \rho= & (x-\xi) d x+(y-\eta) d y+z d z \\
& +n^{\prime}[\eta(x-\xi)-\xi(y-\eta)] d t \\
= & (x-\xi) d x+(y-\eta) d y+z d z \\
& -n^{\prime}[x(y-\eta)-y(x-\xi)] d t .
\end{aligned}
$$

Hence from the equations of motion

$$
\begin{aligned}
& 2\left(\frac{d x}{d t} \frac{d^{2} x}{d t^{2}}+\frac{d y}{d t} \frac{d^{2} y}{d t^{2}}+\frac{d z}{d t} \frac{d^{2} z}{d t^{2}}\right) \\
- & 2 n^{\prime}\left(x \frac{d^{2} y}{d t^{2}}-y \frac{d^{2} x}{d t^{2}}\right) \\
= & -\frac{\phi r}{r} 2\left(x \frac{d x}{d t}+y \frac{d y}{d t}+z \frac{d z}{d t}\right) \\
- & \frac{\psi \rho}{\rho}\left[2\left\{(x-\xi) \frac{d x}{d t}+(y-\eta) \frac{d y}{d t}+z \frac{d z}{d t}\right\}\right. \\
- & \left.2 n^{\prime}\{x(y-\eta)-y(x-\xi)\}\right] .
\end{aligned}
$$

C. VIII. 
But we have

$$
\begin{gathered}
v^{2}=\left(\frac{d x}{d t}\right)^{2}+\left(\frac{d y}{d t}\right)^{2}+\left(\frac{d z}{d t}\right)^{2} \\
x \frac{d^{2} y}{d t}-y \frac{d^{2} x}{d t^{2}}=\frac{d}{d t} r^{2} \frac{d \theta}{d t}=2 \frac{d A}{d t}
\end{gathered}
$$

the foregoing equation may be written

$$
\frac{d \cdot v^{2}}{d t}-4 n^{\prime} \frac{d^{2} A}{d t^{2}}=-2 \phi r \frac{d r}{d t}-2 \psi \rho \frac{d \rho}{d t},
$$

whence

$$
v^{2}-4 n^{\prime} \frac{d A}{d t}=C-2 \int \phi r d r-2 \int \psi \rho d \rho,
$$

the required result.

12. If $x, y$ are the coordinutes of a particle moving in plano under the action of a central force varying as (distance) $)^{-2}$ : write down the expressions of the coordinates $x, y$ in terms of the time $t$ and of four arbitrary constants; and (in case of disturbed motion) starting from the equations

$$
\delta x=0, \delta y=0, \delta x^{\prime}=\frac{d \Omega}{d x} d t, \delta y^{\prime}=\frac{d \Omega}{d y} d t,
$$

(the notation to be explained), indicate the process of finding the variations of the constants in terms of (1) $\frac{d \Omega}{d x}, \frac{d \Omega}{d y}$, (2) the derived functions of $\Omega$ in regard to the constants.

We have

$$
\begin{aligned}
& x=a\left\{\frac{\cos u-e}{1-e \cos u} \cos \varpi+\frac{\sqrt{ }\left(1-e^{2}\right) \sin u}{1-e \cos u} \sin \varpi\right\}, \\
& y=a\left\{\frac{\sqrt{ }\left(1-e^{2}\right) \sin u}{1-e \cos u} \cos \varpi-\frac{\cos u-e}{1-e \cos u} \sin \varpi\right\},
\end{aligned}
$$

where

$$
n-e \sin u=t \sqrt{ }\left(\frac{\mu}{a^{3}}\right)+c,
$$

an equation serving to express $u$ in terms of $t$ and the constants $a, e, c$; the foregoing equations, therefore, in effect give $x, y$ in terms of $t$ and the four constants $a, e, c, \varpi$.

In the second part of the question, $\Omega$ is a given function of $x, y, t$, the differential coefficients $\frac{d \Omega}{d x}, \frac{d \Omega}{d y}$ being the partial ones in regard to $x, y$ respectively. The equation $\delta x=0$ signifies that the variation of $x$, in so far as it arises from the variation of the constants, is $=0$; it in fact means

$$
\frac{d x}{d a} \frac{d a}{d t}+\frac{d x}{d e} \frac{d e}{d t}+\frac{d x}{d c} \frac{d c}{d t}+\frac{d x}{d \varpi} \frac{d \varpi}{d t}=0 .
$$


The value of $x^{\prime}\left(=\frac{d x}{d t}\right)$ is therefore obtained from that of $x$ by differentiating in regard to $t$ alone, as if $a, e, c$, $\sigma$ were constants: viz. $x^{\prime}$ will be a given function of $t, a, e, c, \varpi ; \delta x^{\prime}$ then denotes the variation of $x^{\prime}$ in so far as it arises from the variation of the constants, viz. the equation $\delta x^{\prime}=\frac{d \Omega}{d a} d t$ means

$$
\frac{d x^{\prime}}{d a} \frac{d a}{d t}+\frac{d x^{\prime}}{d e} \frac{d e}{d t}+\frac{d x^{\prime}}{d c} \frac{d c}{d t}+\frac{d x^{\prime}}{d \omega} \frac{d \sigma}{d t}=\frac{d \Omega}{d x} .
$$

There are the like equations in regard to $y, y^{\prime}$, viz. in all, four equations which are linear in regard to $\frac{d a}{d t}, \frac{d e}{d t}, \frac{d c}{d t}, \frac{d \sigma}{d t}$; and which serve to determine these quantities in terms of $\frac{d \Omega}{d x}, \frac{d \Omega}{d y}$.

Now considering the $x, y$ as expressed in terms of $a, e, c, \sigma, t$, then $\Omega$ becomes a function of these quantities; the differential coefficients $\frac{d \Omega}{d a}$, \&c., being connected with the original differential coefficients $\frac{d \Omega}{d x}, \frac{d \Omega}{d y}$ by the equations

$$
\begin{aligned}
& \frac{d \Omega}{d a}=\frac{d \Omega}{d x} \frac{d x}{d a}+\frac{d \Omega}{d y} \frac{d y}{d a}, \\
& \frac{d \Omega}{d e}=\frac{d \Omega}{d x} \frac{d x}{d e}+\frac{d \Omega}{d y} \frac{d y}{d e},
\end{aligned}
$$

\&c.

As there are four equations, $\frac{d \Omega}{d x}, \frac{d \Omega}{d y}$ can be expressed in an infinity of ways in terms of $\frac{d \Omega}{d a}, \frac{d \Omega}{d e}, \frac{d \Omega}{d c}, \frac{d \Omega}{d \sigma}$, and considering $\frac{d a}{d t}$, \&c., as given in terms of $\frac{d \Omega}{d x}, \frac{d \Omega}{d y}$, we can in an infinity of ways express $\frac{d a}{d t}$, \&c., as linear functions of $\frac{d \Omega}{d a}, \frac{d \Omega}{d e}, \frac{d \Omega}{d c}, \frac{d \Omega}{d \boldsymbol{\omega}}$. But there is one form (obtained by combining the equations in a particular manner) wherein the coefficients of the last-mentioned quantities are functions of $a, e, c$, without $t$; and this is the form actually employed for the expression of $\frac{d a}{d t}, \frac{d e}{d t}, \frac{d c}{d t}, \frac{d \varpi}{d t}$ in terms of $\frac{d \Omega}{d a}, \frac{d \Omega}{d e}, \frac{d \Omega}{d c}, \frac{d \Omega}{d \omega}$, in the method wherein these quantities are made use of.

I remark upon the present question, that the answer ought to be in substance perfectly familiar to every student in Physical Astronomy; and that a student ought to be able to present it in a clear and logical form: the question being in fact intended as a test of ability in this respect. 
13. Explain the course of the geodesic lines on a spheroid of revolution: and in particular show that the condition is satisfied in virtue of which any geodesic line, considered as starting from a given point, ceases at some point of its course to be a shortest line.

From each point on the surface a geodesic line may be drawn in any direction whatever along the surface, that is, through each point of the surface there is a singly infinite series of geodesic lines. A geodesic line undulates (in the manner of a sinusoid) between two parallels equidistant from the equator on opposite sides thereof; viz. considering it as starting from a point $A$ on the equator, it arrives at a point $V$ on the upper parallel (there touching the parallel), and passes downwards to cut the equator at $A^{\prime}$, and thence arrives at a point $V^{\prime}$ on the lower parallel (there touching the parallel), and again passes upwards to meet the equator at $A^{\prime \prime}$, and so on; the arcs $A V, V A^{\prime}, A^{\prime} V^{\prime}, V^{\prime} A^{\prime \prime}$, \&c., being similar and equal to each other (differing only in position). The equatoreal arc $A A^{\prime}\left(=A^{\prime} A^{\prime \prime}=\& c\right.$.) or difference of the longitudes $A, A^{\prime}$, is always less than $180^{\circ}$, its value increasing with the inclination at which the geodesic line cuts the equator, viz. when this angle is indefinitely small, the are is $=\frac{c}{a} 180^{\circ} \quad(c, a$ the polar and equatoreal axes respectively), and as the inclination becomes indefinitely near to $90^{\circ}$, the value of the arc becomes indefinitely near $180^{\circ}$. If the arc in question is commensurable with $180^{\circ}$, the geodesic line will be, it is clear, a closed curve; but if not, then it is not a closed curve, but proceeds undulating for ever between the two parallels. In the limiting case where the inclination is $=90^{\circ}$, the geodesic line is obviously a meridian.

Considering a geodesic line starting in a given direction from a point $A$, and the geodesic line from the same point $A$ in the consecutive direction, it appears from the foregoing account of the configuration of the lines, that the two lines will intersect each other in general an indefinite number of times: supposing that they first intersect in a point $K$, then by a general theorem of Jacobi's, the geodesic line $A K$ is a shortest line from $A$ to any point nearer than $K$, but it is not a shortest line from $A$ to any point beyond $K$. 